\title{
Gentle Robotic Handling Using Acceleration Compensation
}

\author{
Suei Jen Chen \\ Universität Karlsruhe (TH) \\ Germany
}

\section{Introduction}

Nowadays, robot manipulators are being widely used in the industrial and manufacturing sector, because of their high precision, speed, endurance and reliability. In the automotive industry, their typical applications include welding, painting, assembly of body, pick and place, etc. Normally, the main task is restricted to simple operations like moving a tool in different working locations. In the sector of logistics, they have to fulfill material handling and storage tasks like palletizing, depalletizing and transportation of goods. Without doubt, the main goal is to maximize the productivity. For this purpose, mostly the robotic manipulators are programmed to drive as fast as possible. But, reaching a time-optimal path does not mean the attainment of a perfect task performance. Since important aspects such as quality and safety should not be overlooked. Usually, most of the handling goods require a special and delicate treatment, due to the object's characteristic and complexity. Otherwise, quality degradations or damages to the transferring object may occur. In the process of material handling, it is common to encounter different kind of problems because of too high accelerated motions. For example: unexpected separation of the transferring object from its grasping tool, the spill-over of fluid materials in highly accelerated liquid containers, such as the transfer of hot molten steel or glass in a casting process, or dangerous collisions caused by swinging suspended objects in harbors or in construction areas, etc.

To overcome the mentioned problems, new efficient approaches based on the so-called Acceleration Compensation Principle will be introduced in this chapter. The main idea is to modify the reference motion trajectory, in order to compensate undesirable disturbances. The position and the orientation of the robot's end-effector are adapted in such a way, that undesired acceleration side effects can be compensated and minimized. In addition, a derivation from the first proposed technique is introduced to compensate undesirable swinging oscillations. Gentle robotic handling and time optimized movements are considered as two main research objectives in the context of this work. Additionally, economic and complexity factor should be also taken into account. Beyond the achievement of optimal cycle time and the avoidance of collisions, the new algorithms are independent of the object's physical aspects and are evaluated with serial robot manipulators, as main part of the experimental test-bed, in order to verify the feasibility and effectiveness of the proposed approaches. 
Since the problems concerning to robotic handling issue could be rather broad, this chapter is limited to analyze and to evaluate three particular problem-scenarios, which are commonly encountered in most industrial applications. Depending on the nature of the handling operation, a fast movement may induce particular collateral effects, such as:

a. Presence of excessive shear forces: undesired sliding effects or loss of transferring objects from the grasping tool, as consequence of too large lateral accelerations.

b. Undesired oscillations in liquid containers: abrupt liquid sloshing and spill-over of liquid materials in highly accelerated open containers, such as molten steel, glass, etc.

c. Swing oscillations in suspended loads: dangerous swing balancing effects in suspended transferring objects, which could induce unexpected collisions.

Based on this classification and before going into the details, a brief overview about the existing methodologies concerned with general robotic handling problems will be introduced in the next section.

\section{State of the Art}

So far, many manufacturers still apply conventional methods to deal with the problems described before. One common and inefficient solution is the reduction of motion accelerations, until the object can be safely transported to the programmed destination. This leads to tedious trial-and-error teaching procedures, and implies also an enormous increase in the cycle time. A further solution is the implementation of active feedback control. In such systems, the controlled parameters are monitored through sensors. Unfortunately, the feasibility of installing sensor devices into the controlling system depends mainly on the properties of the transferring object itself and also on the system infrastructure. Furthermore, most of the sensor systems require extra calibrations and maintenances, which increase enormously the cost and system complexity.

Based on the proposed classification-criteria, important literature surveys dealing with these three problem-scenarios can be seen as follows:

I. Presence of large shear forces: large shear forces can induce undesired sliding effects due to highly accelerated transfer, e.g., transfer of cartons containing fresh fruit or bakery products in the food manufacturing. Here, it is very important that the goods are handled carefully, to maintain the quality of each individual item. The worse scenario is the unwanted losses of goods during the motion. This happens when the resulting forces applied on the handled object have exceeded the maximum permissible. This could cause irremediable damages to the object, the carrying device or/and its surrounding area. A well known example is the transfer of fragile glass cathode ray tubes (CRT) using vacuum suction gripper in the manufacturing of TV.

Dealing with the shear force minimizing, several investigations proposed feedback control system using different kinds of sensor technologies. In [Kolluru et al., 2000], a reconfigurable robotic gripper system was developed to ensure a reliable manipulation of deformable limp material. Later, [Tsourveloudi et al., 2000] incorporated a fuzzy logic control system to regulate the air flow and to ensure safe handling. In this approach, an adequate amount of contact pressure at the gripping points during the entire manipulation process was determined. Other authors dealt with this problem using mobile transportation systems, where the acceleration of the mobile platform is actively compensated to stabilize the transported object, e.g. the well-known selfbalancing mobile robots [Segway, 2004], [Crasser et al., 2002] and [Lauwers et al., 2005]. 
Active Acceleration Compensation technique was firstly proposed by [Graf \& Dillmann, 1997]. In this system, a Stewart Platform was mounted on top of a mobile platform. By tilting this platform, it was possible to compensate the acceleration of the mobile platform in such a way that no shear forces were applied to the transported object. A similar work was established by [Dang et al., 2001, 2002, 2004]. In this case, a parallel platform manipulator was utilized and it tried to emulate a virtual pendulum, with the aim to compensate actively for disturbances in acceleration input. The undesired shear forces were compensated by imitating the behaviour of a pendulum instead of tilting the object.

II. Undesirable oscillations produced by objects with dynamical behaviors: objects with dynamical constraints may suffer oscillation problems during high-speed motions. In this study, relying on the nature of the handling object, the oscillations can be roughly classified into two groups:

a. The first category deals with undesirable sloshing (fluid oscillations) produced by containers with fluid contents. High-speed transfer may cause the spillover of the fluid content and leading to possible contaminations. A popular example is in the casting process, where the transportation of open containers filled with hot molten steel or glass should be executed in high speed and with high positioning accuracy, in order to avoid any undesired cooling of molten material. Hence, it is crucial that such motions are accomplished within a minimum cycle-time and with utmost delicacy, to prevent disturbances produced by undesired forces or oscillations.

Interesting works to be mentioned are the approaches from [Feddema et al., 1996, 1997], which used two command shaping techniques for controlling the surface of a liquid in an open container, as it was being carried by a robot arm. In this work, oscillation and damping of the liquids were predicted using the Boundary Element Method (BEM). Numerous studies dealing with automatic pouring systems in casting industries have been realized. In [Yano \& Terashima, 2001], [Noda et al., 2002, 2004], [Kaneko et al., 2003], the Hybrid Shape Approach was adapted to design an advanced control system for automatic pouring processes. The method introduced by Yano, comprised a suitable nominal model and determined an appropriate reference trajectory, in order to construct a high-speed and robust liquid transfer system, reducing in this way undesired endpoint residual vibrations. The behaviour of sloshing in the liquid container was approximated by a pendulum-type model. A H-Infinity feedback control system was applied.

b. The second category comprises swinging effects caused by suspended objects. These problems are widely encountered in many sectors of industry. These include: operations of cranes on construction site, warehouse, harbor, shipboard handling systems, etc., where the factor 'safety' plays a crucial role.

Several investigations were based on open-loop control technique, in which the concept of optimal control was taken into account [Mita \& Kanai, 1979], [Blazicevic \& Novakovic, 1996] and [Sakawa \& Shindo, 1982]. Most of them used bang-coastbang control, consisting of a sequence of constant acceleration pulses in conjunction with zero acceleration periods. For the control of swing-free boom cranes, Blazicevic adopted a set of velocity basis functions to minimize swing oscillations. The dynamic model of the crane was proposed and transformed into state space for the purpose of examining the dynamic behaviour of a rotary crane, which 
combined speed profiles were employed for the software simulation. In 1957, [Smith, 1957] introduced for first time, a technique which generated a "shaped" input trajectory to the system, with the goal to suppress oscillation effects. The main idea of a command shaper was to excite the system with a sequence of impulses during the entire trajectory, to cancel the perturbing oscillations. This method was later extended by many other researchers [Starr, 1985], [Singer \& Seering, 1990], [Parker et al., 1995] and [Singhose et al., 1997].

As observed, most of the existing studies found in the literature have been proposing either complex modeling of the system or required the help of external sensors for the performance of their proposed controlling methods, at the expense of large efforts, time and money.

In this work, we propose open-loop control methods based on the adaptation of the tool position and orientation. The main aim is to establish a gentle handling of objects without compromising cycle time. With this focusing on the background, the new approaches lead to new robot optimal trajectories, which reduce large lateral accelerations and avoid undesired oscillations acting on the transferring objects, for moving them fast and in a gentle way.

\section{Basic Concept. Acceleration Compensation Principle}

\subsection{Introduction}

Several issues arisen during the handling tasks due to undesired large acceleration effects have been presented in the previous section. Additionally, important scientific-research works dealing with these problems, together with their distinguishing characteristics, were briefly described. As a relevant observation, the majority of these methods require either additional sensors or an accurate model of the system, which consequently imply major computational effort and algorithm complexity. As we observe, problems with material handling may occur in different ways, in which each issue require a careful analysis and an individual solution. The main contribution of this investigation is to provide generalized solutions, which are simple, time-efficient and feasible to solve handling problems.

Considering the nonlinearity of the system and the conservation of product's quality without overlooking the safety and the cost reducing, Acceleration Compensation Principle $(\mathrm{ACP})$ is considered as an appropriate solution, offering great efficiency and robustness. Based fundamentally on the approach proposed by [Graf \& Dillmann, 1999, 2001], our system employs mainly robot manipulators as transferring devices, which move and tilt their carrying tool in such a way, that no undesired accelerations effects could exist, to affect the current state of the transporting object.

\subsection{The "Waiter-Tray" Model}

This idea was born by observing humans carrying objects (those which need special care and attention) rapidly from one location to another. A good example is a waiter walking inside a restaurant holding up with his hand a tray full of plates and glasses, without throwing away the transported elements. Probably, without knowing it, the waiter tries to incline the tray in such a way that unwanted accelerations and forces acting on the carried objects are avoided. The main approach presented in this work has a similar mechanism: while the waiter is orienting his hand to tilt the tray in an appropriate manner, the 
orientation of the robot's end-effector is adapted as well to compensate for undesired acceleration effects [Fig. 1].
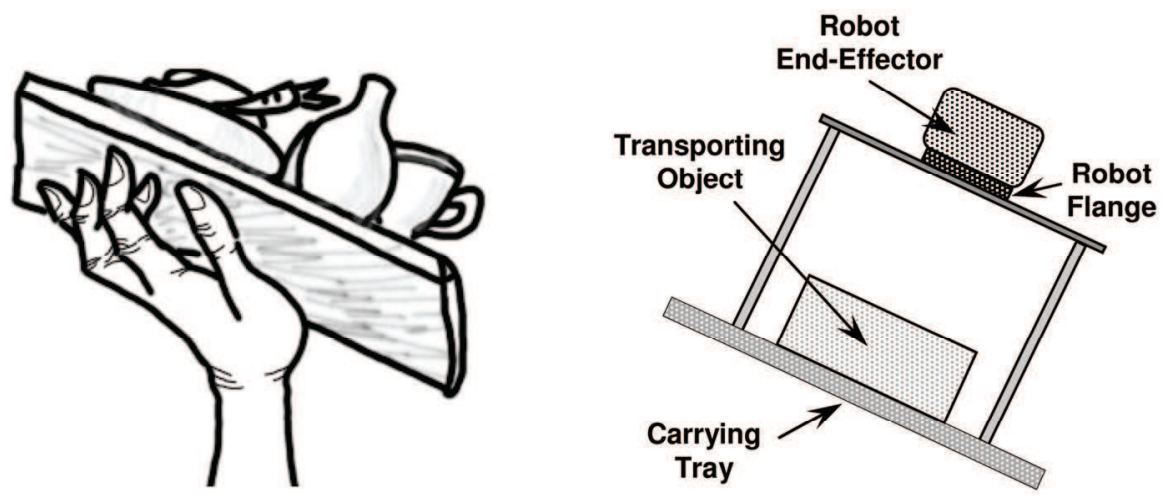

Figure 1. Robot arm imitating a human hand to carry objects

\subsection{The Test-Environment}

According to the problem-scenarios described before, three test-beds consisting of a robot manipulator with different kind of tools, which functionally replicate the transfer system for testing, are used to verify the effectiveness of the proposed approaches [Fig.2].

\section{Problem-Scenario I: Reduction of Shear Forces}

\subsection{Introduction}

Commonly, the handling robots employ vacuum suction grippers as gripping tool to manipulate and to move goods from one place to another. Although vacuum suction grippers have a lot of advantages, there is still one significant drawback: due to the phenomenon of large lateral accelerations, the highly accelerated load could fall off the gripper. To solve this problem, the orientation of the robot's end-effector is adapted in such a way, that the undesired large shear forces, between the contact surface of the grasping tool and its carrying object, can be minimized until the end of the motion.
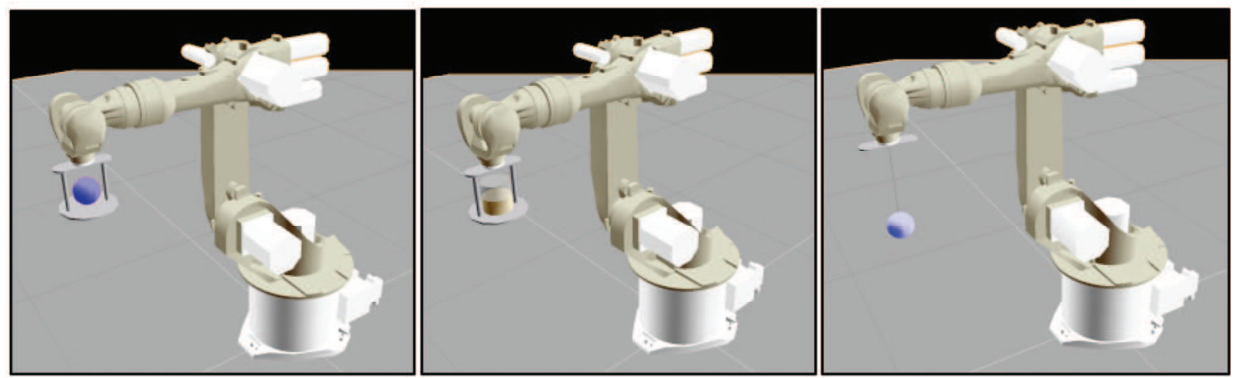

Figure 2. Three problem-scenarios in evaluation. Minimizing of shear forces (a), liquid sloshing (b) and sway oscillations (c) 


\subsection{Model Description}

With the need to describe the problem in question, and to observe the effects created by large shear forces, a simplified model using a tray is proposed. Fig. 3 shows a simplified free-body diagram with the principal forces involved in such a system. $F_{\text {sh }}$, indicates the shear force. $F_{f}$ is the friction force, it resists to any force that tries to move an object along a surface and it acts oppositely to the shear force, thus $F_{f}=-F_{s h}$, as long as the object is not slipping. $F_{N}$ is the normal force that the tray exerts on the transporting object, it remains perpendicular to the tray's surface, $g$ is the gravitational acceleration and thus, $m g$ represents the object's weight.

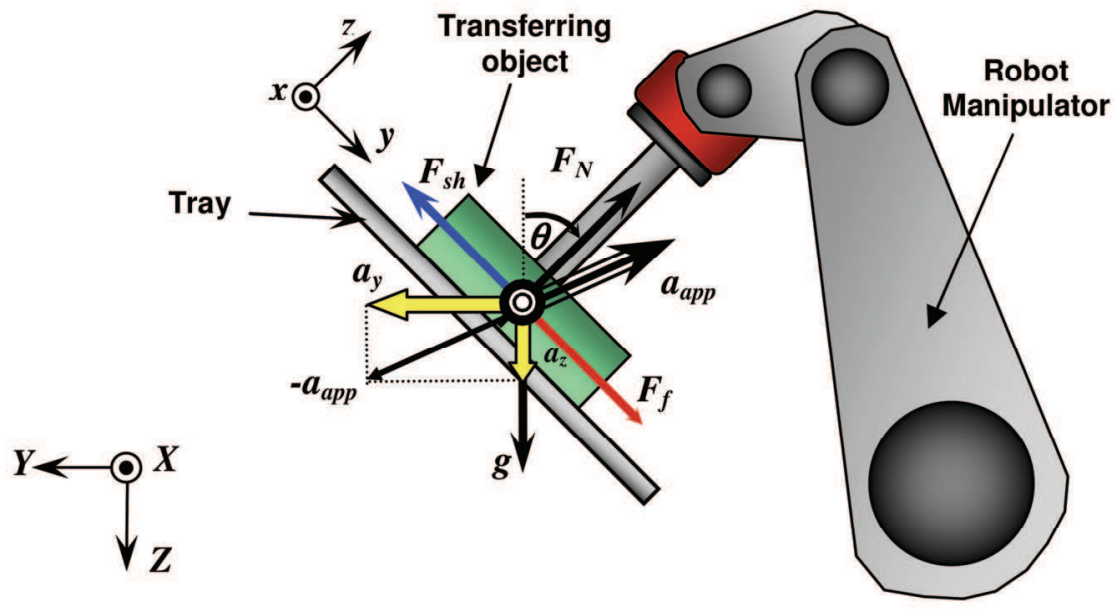

Figure 3. Free body diagram. Robot manipulator with the transferring object

$a_{a p p}$ represents the total acceleration applied by the end-effector, it points in the direction of the motion. $a_{x}, a_{y}$ and $a_{z}$ are the inertial accelerations opposed to any acceleration acting on the object in $x-, y$ - and $z$-direction respectively, due to horizontal/vertical motion of the endeffector (relative to the $X-Y-Z$ world coordinate system), $x, y$ and $z$ establish the reference frame system in the tray. $\theta$ symbolizes the tilting angle of the TCP(Tool Center Point).

The fundamental requisite to guarantee that the carried object remains on spot in the carrying tray is, that the magnitude of the shear force must be less than or equal to the magnitude of the maximum friction force:

$$
\left|F_{s h}\right| \leq\left|F_{f_{\max }}\right| \text {. }
$$

This is a very important condition, which has to be taken into account. The proposed method works when this inequation is fulfilled. It is also important to notice that in an ideal case, the optimal value of the shear force is considered as zero.

\subsection{Optimal Tilting Angles}

Now, considering that all forces acting on the object are referring to the tray reference coordinate system. The forces along the contact surface of the object in $y$-direction $(x-$ direction can be treated in the same way) is defined as: 


$$
F_{s h_{y}}=m\left(g \sin \left(\theta_{y}\right)+a_{z} \sin \left(\theta_{y}\right)-a_{y} \cos \left(\theta_{y}\right)\right) .
$$

This yields to the definition of the shear force. To achieve the condition (1), a change in the orientation of the TCP is needed. This guarantees that the shear force $F_{s h}$ is minimized in every time-instance. Hence, deriving from (2) and assuming the "ideal case", where the shear force is zero, the mass $m$ can be eliminated. This means that the role of the mass for the new approach is irrelevant and this gives:

$$
\left(g+a_{z}\right) \sin \left(\theta_{y}\right)-a_{y} \cos \left(\theta_{y}\right)=0 .
$$

Thus, the value of $\theta_{y}$ can be computed and as well in an analogue way for $\boldsymbol{\theta}_{x}$. These are the optimal tilting angles due to $y$ - and $x$-horizontal movement respectively. Note that the tilting angles are functions of each time-instance $t$ :

$$
\begin{aligned}
& \theta_{x}(t)=\tan ^{-1}\left(\frac{a_{x}(t)}{\left(g+a_{z}(t)\right)}\right), \\
& \theta_{y}(t)=\tan ^{-1}\left(\frac{a_{y}(t)}{\left(g+a_{z}(t)\right)}\right) .
\end{aligned}
$$

During the motion, if the robot controller adapts its tool orientation according to (4) and (5), the load is guaranteed to be kept on spot in the carrying tray.

\subsection{Lateral Acceleration}

Once that the definition of shear force has been introduced, the lateral acceleration $a_{\text {lat }}$ can be determined according to (2) and it is expressed as follows

$$
a_{\text {lat }}=F_{s h_{y}} / m=g \sin \left(\theta_{y}\right)+a_{z} \sin \left(\theta_{y}\right)-a_{y} \cos \left(\theta_{y}\right),
$$

$a_{\text {lat }}$ is the lateral acceleration, consequence of the remaining shear force. Let's consider the model described in Fig. 3. In case of no existence of tilting movement (that is $\boldsymbol{\theta}_{y}=0$ ), then the lateral acceleration is equivalent to $a_{l a t_{y}}=-a_{y}$ until the end of motion. This means, when no compensation is executed, then the lateral acceleration acting on the transfer object has the same magnitude but opposite to the original applied acceleration. In this situation, if the friction is not large enough, the object will easily fall down from the carrying tray.

\subsection{Motion Feasibility}

As seen in (4) and (5), a fast increasing of acceleration will lead to fast changes in the tilting angles. Because modern robots are highly dynamic machines, the acceleration is ramped up very fast to the maximum (trapezoidal motion profile for the velocity) leading to high jerks. This fast change of orientation can not be achieved with a standard commercial robot, because of severe dynamic performance limits, such as maximum motor torque and maximum gear load. In order to avoid too large jerk effects and therefore to achieve suitable motions, one possible solution is the modification of shape of velocity and acceleration 
profile using filtering algorithms, with the aim to reach smoother motions during the periods of acceleration and deceleration [Chen et al., 2006].

\subsection{Simulation and Experimental Results}

After analyzing theoretically the lateral acceleration, a linear motion is first put into examination using software simulation, which its results and performance are later evaluated through a virtual robot controller, in order to verify its feasibility before putting the motion into the test environment with real robot manipulators. Here, the results are also verified though the measurements using an axis accelerometer, a development kit ADSL202 from ANALOG DEVICES with a measurement range of $\pm 2 \mathrm{~g}$.

\section{A. Test Setup}

To simplify the simulation analysis, a simple linear motion along $Y$-axis is evaluated. The trajectory begins at the starting position $\mathbf{A}=[0.370,0.300,0.390]$ and ends at position $\mathbf{B}=$ $[0.370,-0.300,0.390][\mathrm{m}]$. The positions are expressed in Cartesian coordinate system [Fig. 4]. Every interpolation cycle $t_{i p o}$ is $0.012 \mathrm{~s}$. The maximum acceleration for continuous motions is set to $4.6 \mathrm{~m} / \mathrm{s}^{2}$ and maximum velocity to $2 \mathrm{~m} / \mathrm{s}$.

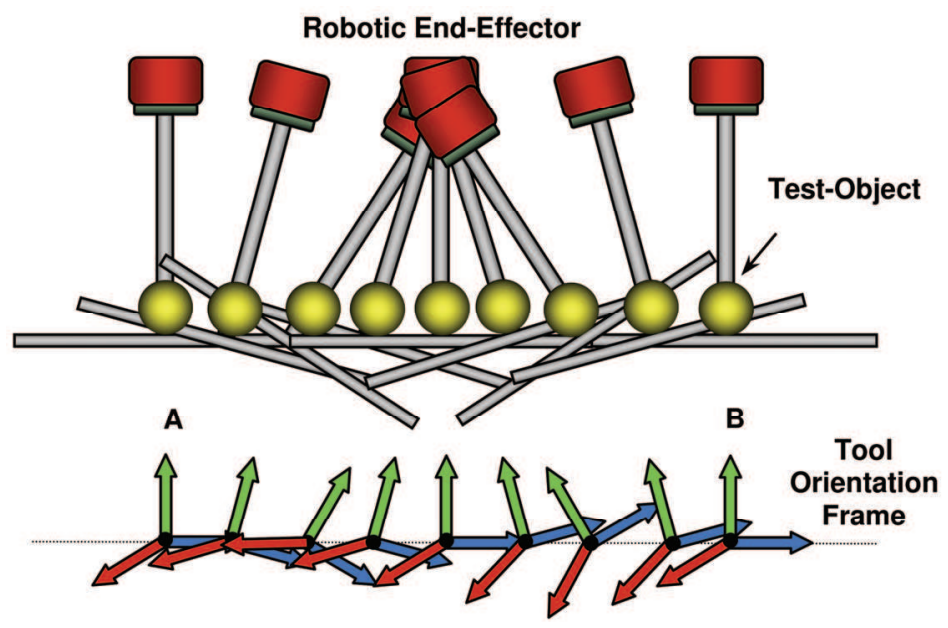

Figure 4. Trajectory of robot TCP with compensated tilting angles

\section{B. Results}

Using simulation software, the above motion is tested with diverse filter lengths $L=9,15$ and 30 [Fig. 5]. In this particular case, the reference acceleration requires a total of $0.816 \mathrm{~s}$ to complete the programmed movement. The best compensated motion is when the filter length $L=9$, with a filtered lateral acceleration (maximum absolute) of $2.1 \mathrm{~m} / \mathrm{s}^{2}$ and a cycle time of $1.032 \mathrm{~s}$. When $L<9$, the adaptation of the TCP-orientation can not be accomplished due to the robot dynamic limits. Thus $L=9$ is the best suitable filter length. The measurement results can be observed in [Fig. 6].

It is worth to mention that there is a difference between the simulated and the measured curves, due to the fact that the sensor signals themselves have to be filtered to be useful (filter length of sensor $=20 t i_{p o}$, where $t i_{p o}$ represents the robot controller's interpolation cycle 
time). Taking this fact into account, the experimental results confirm satisfactorily the simulation results.

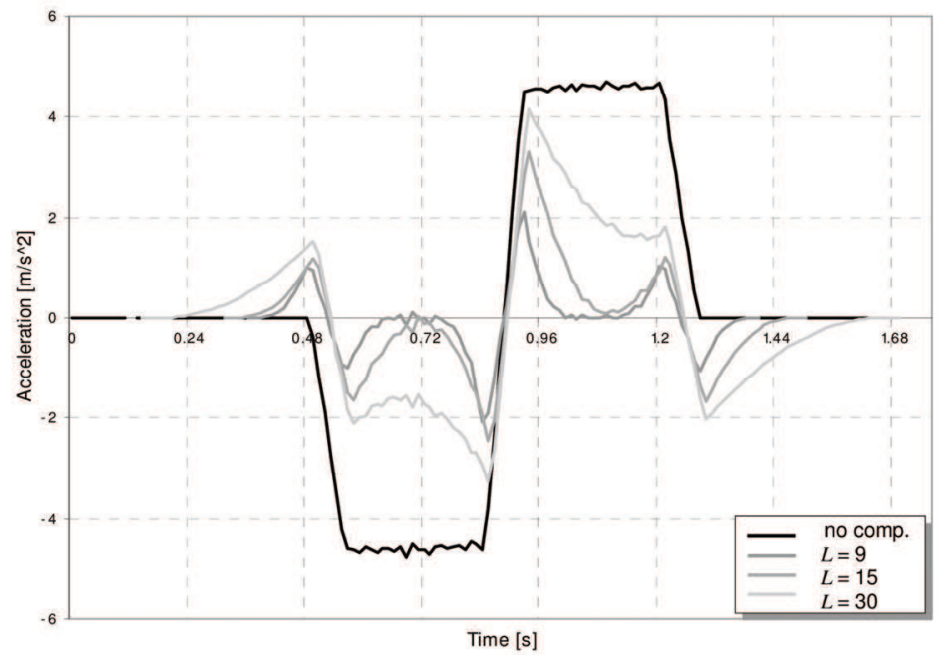

Figure 5. Lateral acceleration with different filter lengths in the software simulation

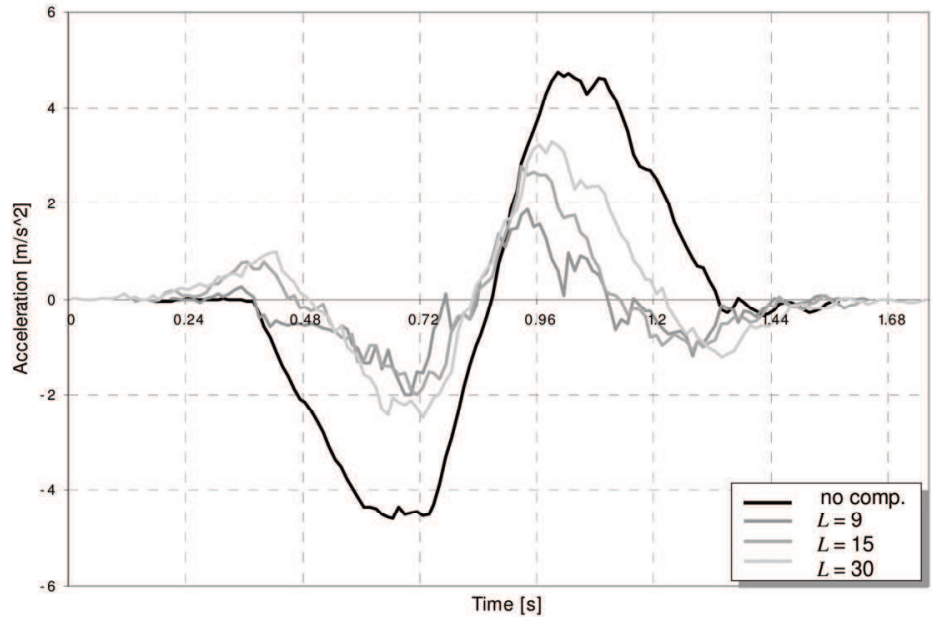

Figure 6. Measurements of lateral acceleration with different filter lengths

\section{Problem-Scenario II: Sloshing Suppression}

\subsection{Introduction}

In the last decades, the topic of suppressing the sloshing or liquid vibration has become a great interest to many sectors, especially in the areas of industry and research. The prevention and avoidance of overflow caused by sloshing is fundamental for many industrial applications, which include liquid container transfer systems. In such systems, 
like the transportation of molten metal from a furnace [Yano \& Terashima, 2001], [Hamaguchi et al., 1994], [Feddema et al., 1997], automatic pouring system in the casting industries [Terashima \& Yano, 2001], [Linsay, 1983], [Lerner \& Laukhin, 2000] or transfer operations with fluid products in the food industry, etc. Here, the sloshing suppression is essential, in order to prevent any negative effect on the product's quality and as well to avoid any possible contaminations.

\subsection{Acceleration Compensation "against" Sloshing?}

Now, a difficult and even more challenging handling problem is introduced. Compared to the manipulation of rigid objects as described in the preceding section, the fluid behaviour is much more difficult to be modelled and controlled, due to its nonlinear dynamical characteristics. Here, any disturbance may cause its deformation and hence, the producing of instabilities to the entire system.

Theoretically, as long as there is no relative motion between the liquid and the container, then the liquid is assumed as a rigid-body. In this case, ACP may be also suitable and applicable to solve the sloshing problems. As main idea, this method operates basically in maintaining the normal of the liquid surface to be opposite to the accelerations of the entire system, until the completion of the transportation [Fig. 7]. In this way, undesired sloshing effects could be enormously reduced, which assures that the liquid can stay safely inside of its container during the entire motion process.

(a)

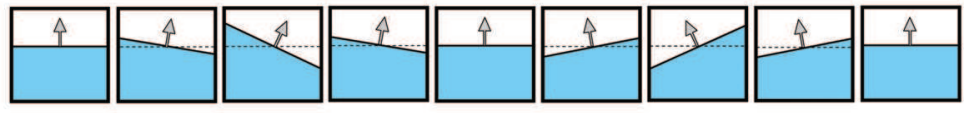

(b)

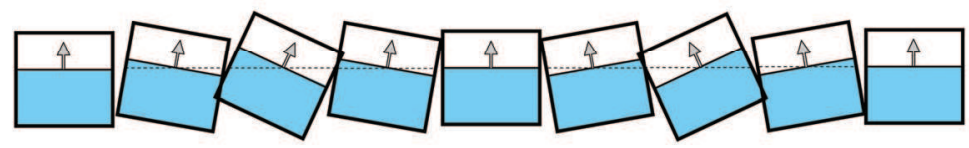

Figure 7. Trajectory of a rectangular liquid container without (a) and with (b) compensated tilting angles

Next, with the aim to validate the proposed theory, important fluid dynamic fundamentals will be introduced, in order to deduce the optimal tilting angles required to compensate the sloshing effects inside an accelerated liquid container.

\subsection{Model Description}

First, an open container of liquid is considered. It translates along a straight path with a constant acceleration $\vec{a}$ as illustrated in Fig. 8. Since $a_{x}=0$, the pressure gradient in the $x$ direction is zero $(\partial p / \partial x=0)$ [Chen et al., 2006]. In the $y$ - and $z$-directions, it yields

$$
\begin{aligned}
-\frac{\partial p}{\partial y} & =\rho a_{y} \\
\frac{\partial p}{\partial z} & =-\rho\left(g+a_{z}\right)
\end{aligned} .
$$


Now, the variation in pressure between two closely spaced points located at $y$ and $z$ is considered. Thus, $y+d y$ and $z+d z$ can be expressed as

$$
d p=\frac{\partial p}{\partial y} d y+\frac{\partial p}{\partial z} d z .
$$

Using the results obtained from Eq. (7) and (8),

$$
d p=-\rho a_{y} d y-\rho\left(g+a_{z}\right) d z .
$$

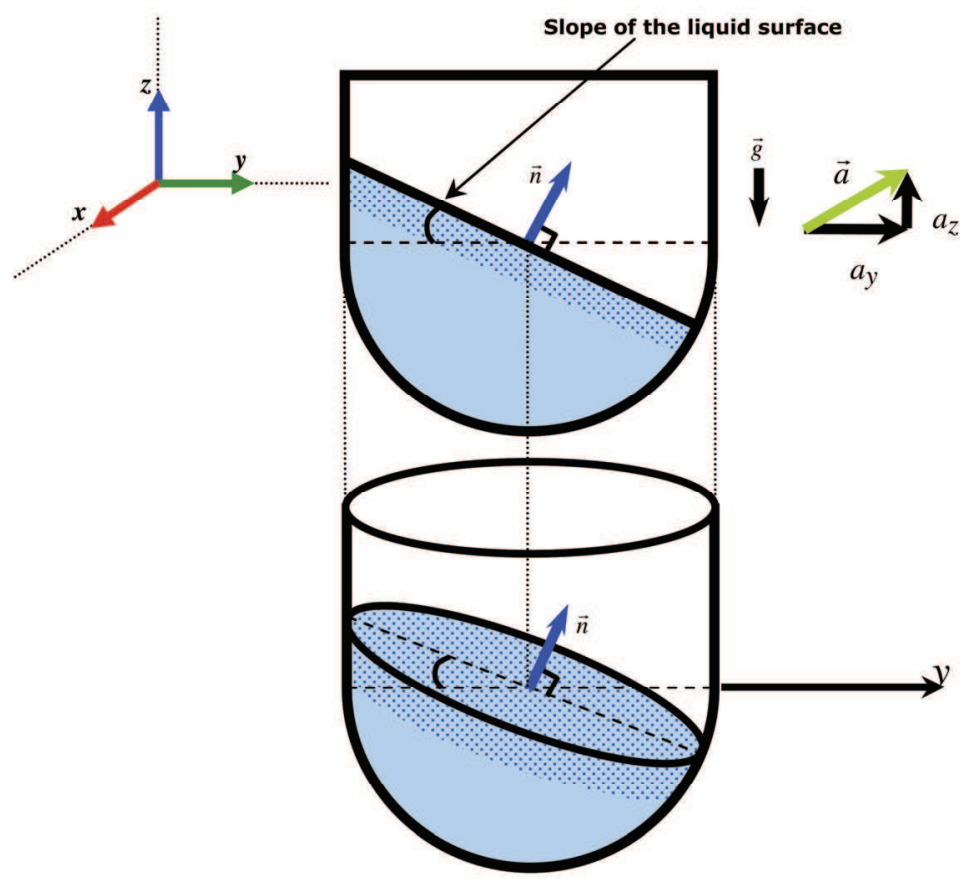

Figure 8. Free body diagram. An accelerating fluid container without compensation

\subsection{Optimal Tilting Angles}

Now, if the pressure is considered constant, then $d p=0$. According to Eq. (9), it follows that the slope of the liquid surface is given by the relationship

$$
\frac{d z}{d y}=-\frac{a_{y}}{\left(g+a_{z}\right)},
$$

where $d z / d y$ is equivalent to $\tan \left(\theta_{y}\right)$. Therefore, it gives

$$
\theta_{y}=\tan ^{-1}\left(\frac{a_{y}}{\left(g+a_{z}\right)}\right),
$$

the same conclusion as obtained in Eq. (4). 


\subsection{Simulation and Experimental Results}

For the verification, a test-bed consisting of a KUKA KR16 industrial manipulator with 6DOF (Degrees Of Freedom) and a metal tray as carrying tool has been used. A camera was adopted as sensor apparatus to observe the liquid behaviour in 2D, and it has been attached directly on the experimental tray, opposing to the glass-recipient.

\section{A. Test Setup}

For the evaluation, two motions are carried out: one motion without, and the other one, with acceleration compensation. As test motion, a linear motion along the $\mathrm{Y}$-axis is used. It starts at the position $\mathbf{A}=\left[\begin{array}{lll}0.930 & 0.800 & 1.012\end{array}\right]$ and ends at the position $\mathbf{B}=\left[\begin{array}{l}0.930 \\ -0.8001 .012\end{array}\right]$.

\section{B. Results}

As test object, a transparent rectangular glass-recipient containing water is used. The static liquid level is $40 \mathrm{~mm}$. The maximum acceleration for continuous motions is set to $4.3 \mathrm{~m} / \mathrm{s}^{2}$. The sequences of the filtered images, obtained from the experimentation-videos, verify that the sloshing effects can be diminished significantly after applying the compensation algorithm [Fig. 9]. For such compensated movement, the adopted filter length is $L=9$.

Here, some residual oscillations still exist slightly during and at the end of the motion, as a consequence of the phase-delay generated after the filtering [Chen et al., 2007]. This oscillation effect is caused because of the differences between the original and the filtered motion. In the case of the non-compensated motion, the maximum deviation of the peak elevation is approximately $37.5 \mathrm{~mm}$ respect to the corresponding static level [Fig. 10]. Contrary to this, the compensated motion has only a maximum deviation around $5.7 \mathrm{~mm}$ at its fluid surface. This represents a reduction of approximately $84.7 \%$.

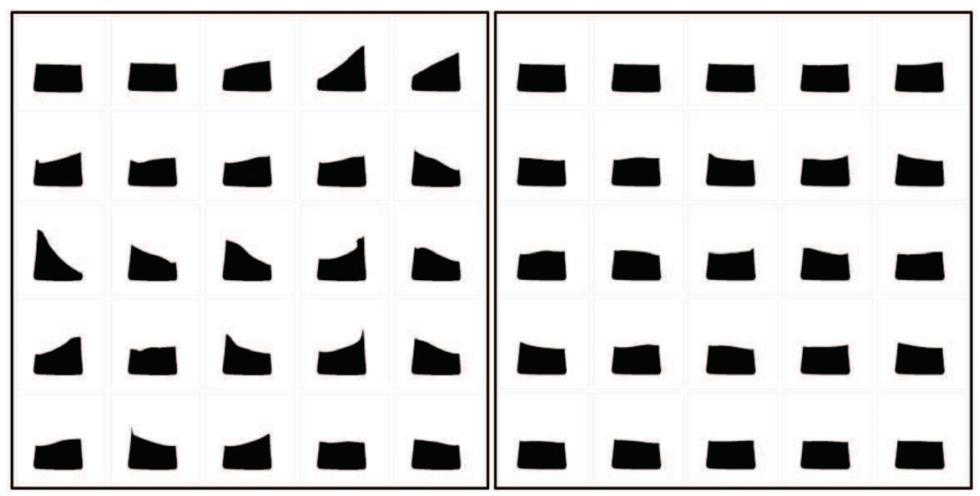

(a)

(b)

Figure 9. Part of image sequences from a linear movement without (a) and with (b) acceleration compensation. The sequence order follows from left to right and from top to bottom

Notice that in the compensated motion, the liquid surface reestablishes its resting state faster than in the non-compensated case. The maximum amplitude of those oscillations is below 6 $\mathrm{mm}$, which assures that the liquid can stay safely inside of its container during the entire motion process. 
This approach is very efficient, since it neither requires any complex fluid modelling, nor the help of an external sophisticated sensing system, or the feedback of any vibration information.
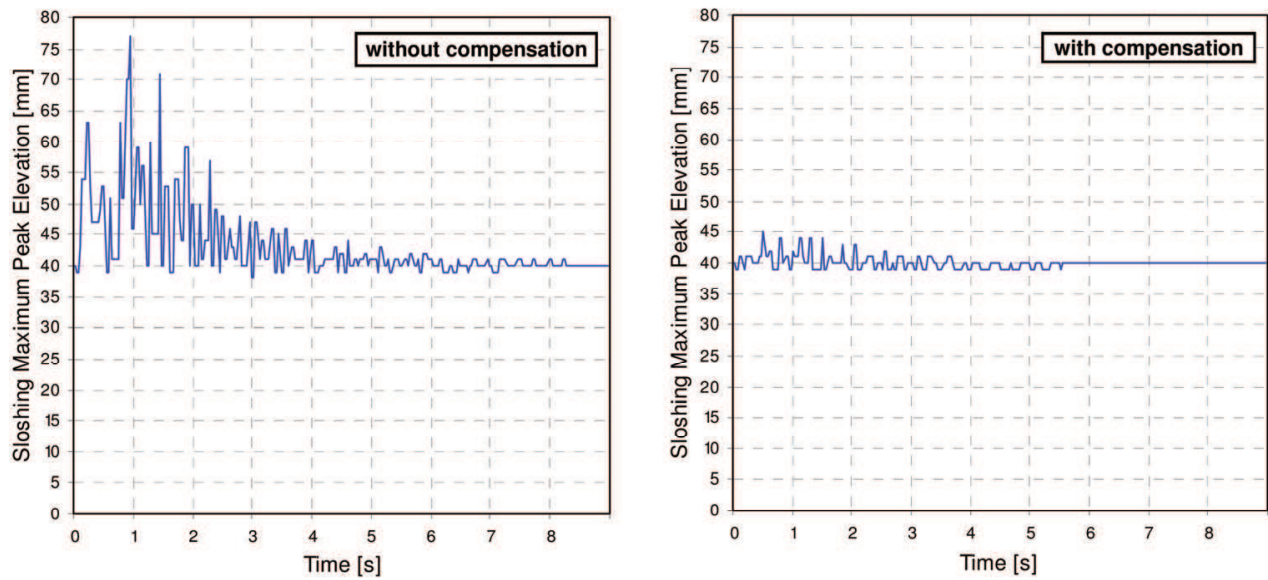

Figure 10. Non-compensation versus compensation. Results obtained from the sensorcamera images

\section{Problem-Scenario III: Minimization of Swing Oscillation}

\subsection{Introduction}

As we mentioned before, high-speed transportation of loads using rope may cause undesired swing oscillations. Next, with the purpose to solve this problem in a simple way, a new open-loop technique is proposed. It derives immediately from the ACP and consists mainly on modifying the reference-trajectory (which is comprised by a set of suspension points) in such a way, that any undesirable swing effects arisen during the motion execution can be suppressed without the help of any external sensor system.

\subsection{The New Compensation Strategy}

This new technique is as a result of the model proposed in Section 4. In this former method, the orientation of robot's end-effector (optimal tilting angles) was remodified according to the compensation algorithm, in order to minimize and compensate undesired acceleration side effects.

On the basis of this principle, our new methodology uses mainly the computed optimal tilting angles to calculate a new compensated trajectory, composed by a new set of suspension points. This means, based on the ACP, the reference input trajectory is modified in such a way, that the resulting movements from the robot's TCP permit the minimizing of undesired swing effects caused by large motion accelerations. Referring to the model described in [Fig. 3], a robot manipulator with a carrying tool is reconsidered [Fig. 11] and it performs a linear horizontal motion from position $\mathbf{A}$ to $\mathbf{B}$.

The optimal tilting angles $\boldsymbol{\theta}_{x}$ and $\boldsymbol{\theta}_{y}$ are defined as indicated in Eq. (4) and (5). As shown in Fig. 11, each computed optimal tilting angle is comprised between the axis of the tool and 
the vertical plane. Additionally, the turning point is referred to the programmed TCP. In this case, the TCP locates at the center of the imaginary tool.

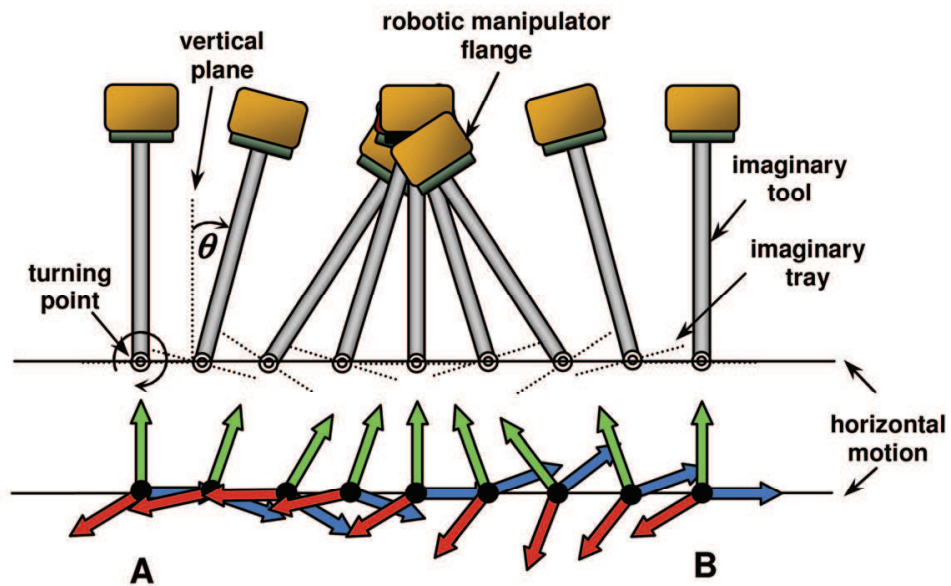

Figure 11. Former acceleration compensation model proposed in section 4

\subsection{A New Set of Suspension Points. Model Description}

A pendulum model is proposed [Chen et al., 2007]. An object with mass $m$ is connected to a robot manipulator through a rigid rope with invariant length $l$ [Fig. 12].

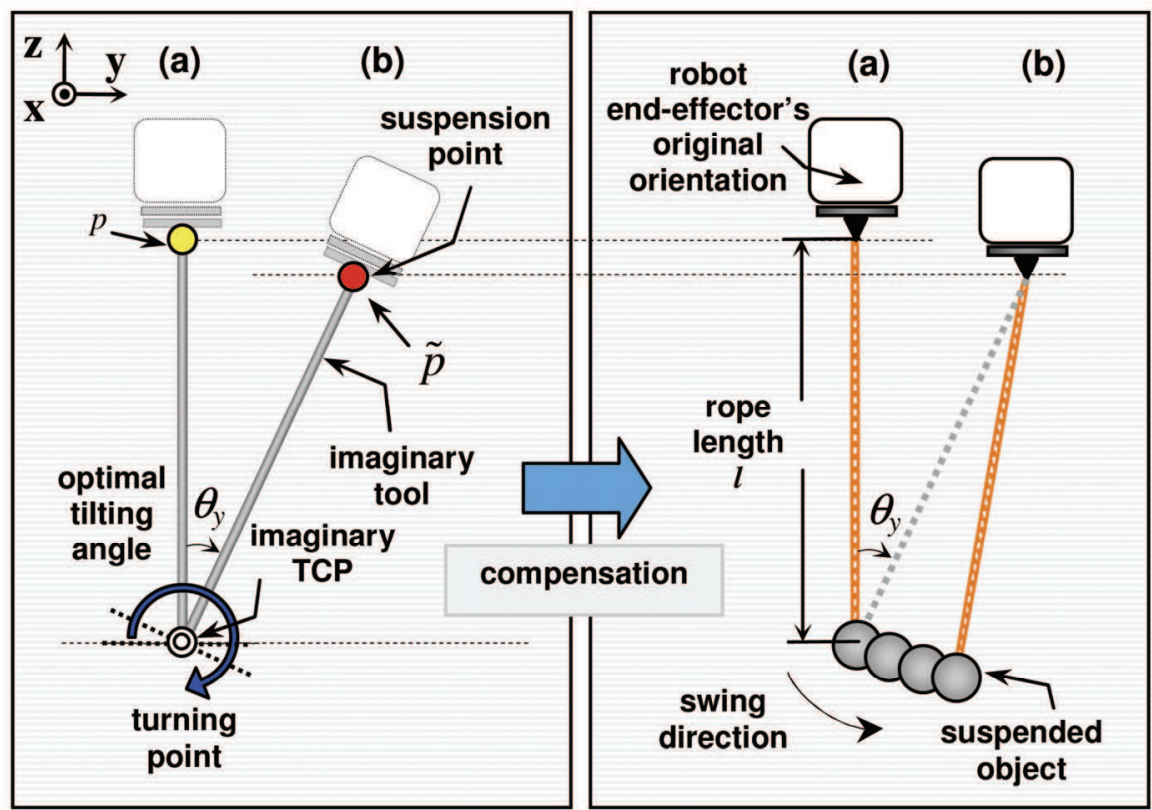

Figure 12. Left: the "imaginary tool" rotates its "imaginary TCP" according to the optimal tilting angle $\boldsymbol{\theta}_{y}$. Right: new compensated trajectory with the suspended object 
The suspension point is attached directly to the robot flange, and the friction due to the bending of the rope is neglected. To compute the new compensated trajectory, the existence of an "imaginary tool" with the same length $l$ as the rigid rope is assumed [Fig. 12(left)]. At its lower endpoint, where theoretically locates the suspended object, an "imaginary TCP" (the turning point) is considered. Now, the robot end-effector moves horizontally to the right [Fig. 12 (left) from (a) to (b)], with the initial conditions $v(0)=0$ and $a(0)=0 . p$ is a Cartesian position from the reference input trajectory, $v$ and $a$ are respectively the rate of displacement (velocity) and rate of velocity variation with respect to time (acceleration).

In order to compute the new compensated trajectory, which is composed by a set of new suspension points of the pendulum, the set of $\tilde{p}$ [Fig. 12 (left)] is calculated with the help of a rotation matrix (Eq. 12). Together with the information of the rope length $l$, a rotation in the turning point is performed, according to $\theta_{x}$ and $\theta_{y}$. To specify the orientation, the convention roll, pitch and yaw angles are used. The rotation around axis $x$ is represented by $\boldsymbol{\theta}_{x}$ (roll), rotation about $y$ by $\boldsymbol{\theta}_{y}$ (pitch), and around axis $z$ by $\boldsymbol{\theta}_{z}$ (yaw).

$$
\begin{aligned}
& R_{r p y}\left(\theta_{x}, \theta_{y}, \theta_{z}\right)=\operatorname{Rot}\left(Z, \theta_{z}\right) \operatorname{Rot}\left(Y, \theta_{y}\right) \operatorname{Rot}\left(X, \theta_{x}\right) \\
& =\left[\begin{array}{ccc}
\mathrm{c}_{z} & -\mathrm{s}_{z} & 0 \\
\mathrm{~s}_{z} & \mathrm{c}_{z} & 0 \\
0 & 0 & 1
\end{array}\right]\left[\begin{array}{ccc}
\mathrm{c}_{y} & 0 & \mathrm{~s}_{y} \\
0 & 1 & 0 \\
-\mathrm{s}_{y} & 0 & \mathrm{c}_{y}
\end{array}\right]\left[\begin{array}{ccc}
1 & 0 & 0 \\
0 & \mathrm{c}_{x} & -\mathrm{s}_{x} \\
0 & \mathrm{~s}_{x} & \mathrm{c}_{x}
\end{array}\right], \\
& =\left[\begin{array}{ccc}
\mathrm{c}_{z} \mathrm{c}_{y} & \mathrm{c}_{z} \mathrm{~s}_{y} \mathrm{~s}_{x}-\mathrm{s}_{z} \mathrm{c}_{x} & \mathrm{c}_{z} \mathrm{~s}_{y} \mathrm{c}_{x}+\mathrm{s}_{z} \mathrm{~s}_{x} \\
\mathrm{~s}_{z} \mathrm{c}_{y} & \mathrm{~s}_{z} \mathrm{~s}_{y} \mathrm{~s}_{x}+\mathrm{c}_{z} \mathrm{c}_{x} & \mathrm{~s}_{z} \mathrm{~s}_{y} \mathrm{c}_{x}-\mathrm{c}_{z} \mathrm{~s}_{x} \\
-\mathrm{s}_{y} & \mathrm{c}_{y} \mathrm{~s}_{x} & \mathrm{c}_{y} \mathrm{c}_{x}
\end{array}\right]
\end{aligned}
$$

where $\mathrm{c}_{x}=\cos \theta_{x}, \mathrm{c}_{y}=\cos \theta_{y}, \mathrm{c}_{z}=\cos \theta_{z}, \mathrm{~s}_{x}=\sin \theta_{x}, \mathrm{~s}_{y}=\sin \theta_{y}$ and $\mathrm{s}_{z}=\sin \boldsymbol{\theta}_{z}$. Now using this rotation matrix, the new suspended point $\tilde{p}$ is computed. Considering that there is no rotation around $Z$-axis, then $\boldsymbol{\theta}_{z}=0$. The position $\tilde{p}$ is defined as

$$
\left[\begin{array}{c}
\tilde{p}_{x} \\
\tilde{p}_{y} \\
\tilde{p}_{z}
\end{array}\right]=\left[\begin{array}{c}
p_{x} \\
p_{y} \\
p_{z}-l
\end{array}\right]+\left[\begin{array}{ccc}
\mathrm{c}_{y} & \mathrm{~s}_{y} \mathrm{~s}_{x} & \mathrm{~s}_{y} \mathrm{c}_{x} \\
0 & \mathrm{c}_{x} & -\mathrm{s}_{x} \\
-\mathrm{s}_{y} & \mathrm{c}_{y} \mathrm{~s}_{x} & \mathrm{c}_{y} \mathrm{c}_{x}
\end{array}\right]\left[\begin{array}{l}
0 \\
0 \\
l
\end{array}\right]=\left[\begin{array}{c}
p_{x}+l \mathrm{~s}_{y} \mathrm{c}_{x} \\
p_{y}-l \mathrm{~s}_{x} \\
p_{z}+l \mathrm{c}_{y} \mathrm{c}_{x}-l
\end{array}\right],
$$

then, the new suspension point is given by

$$
\begin{aligned}
& \tilde{p}_{x}=p_{x}+l \sin \theta_{y} \cos \theta_{x} \\
& \tilde{p}_{y}=p_{y}-l \sin \theta_{x} \\
& \tilde{p}_{z}=p_{z}+l \cos \theta_{y} \cos \theta_{x}-l
\end{aligned}
$$

This is the new position for the suspension point $\tilde{p}$ at the time instant $i$. The set of "old" suspension points are shifted in such a way, that possible rest swing oscillations induced by 
a high-speed motion can be reduced enormously by applying the acceleration compensation method.

\subsection{Compensation vs. Non-Compensation}

To evaluate the shape of the resulting trajectory established by the new suspension points, two examples are illustrated in Fig. 13.

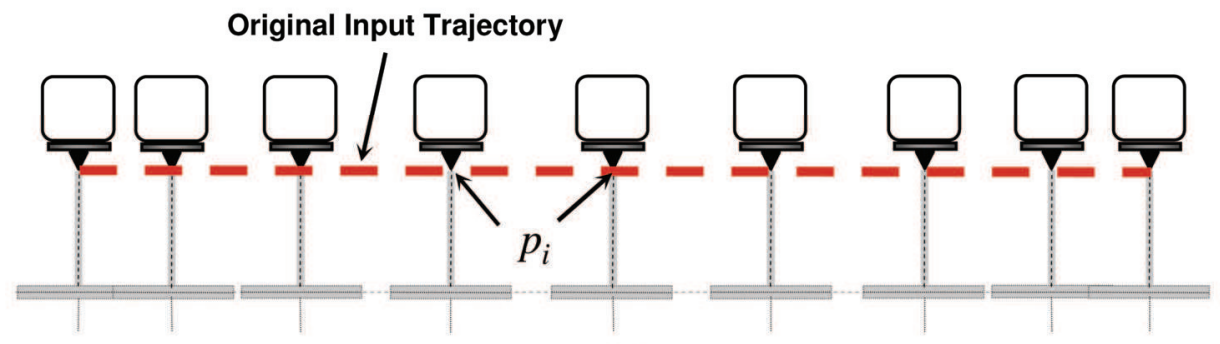

(a)

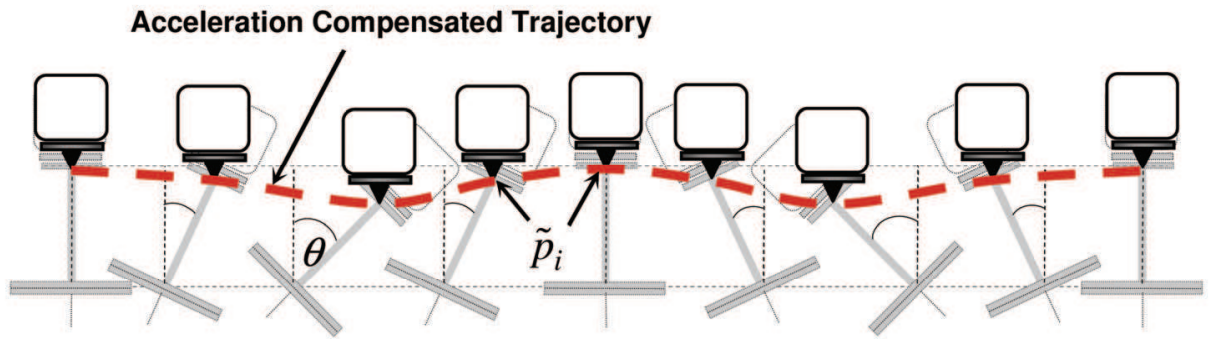

(b)

Figure 13 (a) Trajectory established by the original suspension points, (b) New trajectory applying acceleration compensation

The original trajectory without applying acceleration compensation technique remains a straight horizontal trajectory. On the other hand, the location of each suspension point from a compensated motion, involves not only motions in horizontal-plane, but also vertical movements to eliminate the oscillation effects. Two motions with different lengths are shown in Fig. 14. As observed, the short motion in a reduced time term requires "more movements" to compensate the oscillations.

\subsection{Software Simulation}

Since the swing effect relies principally on the driving accelerations, it is interesting to analyze its behaviour before and after the compensation algorithm.

For simplicity, a test-motion translates in horizontal $Y$-direction. The utilized rigid rope has a length of $0.340 \mathrm{~m}$. Since the reference test motion is a linear continuous-path $(\mathrm{CP})$, the maximum velocity is set to $2 \mathrm{~m} / \mathrm{s}$. The resulting velocity and acceleration profiles before and after applying the compensation algorithm are illustrated in Fig. 15 respectively. All illustrations on the left side of each figure belong to the reference motion without acceleration compensation and on the right side is the compensated motion. The resulting swing angles of both motions are shown in Fig. 16. 

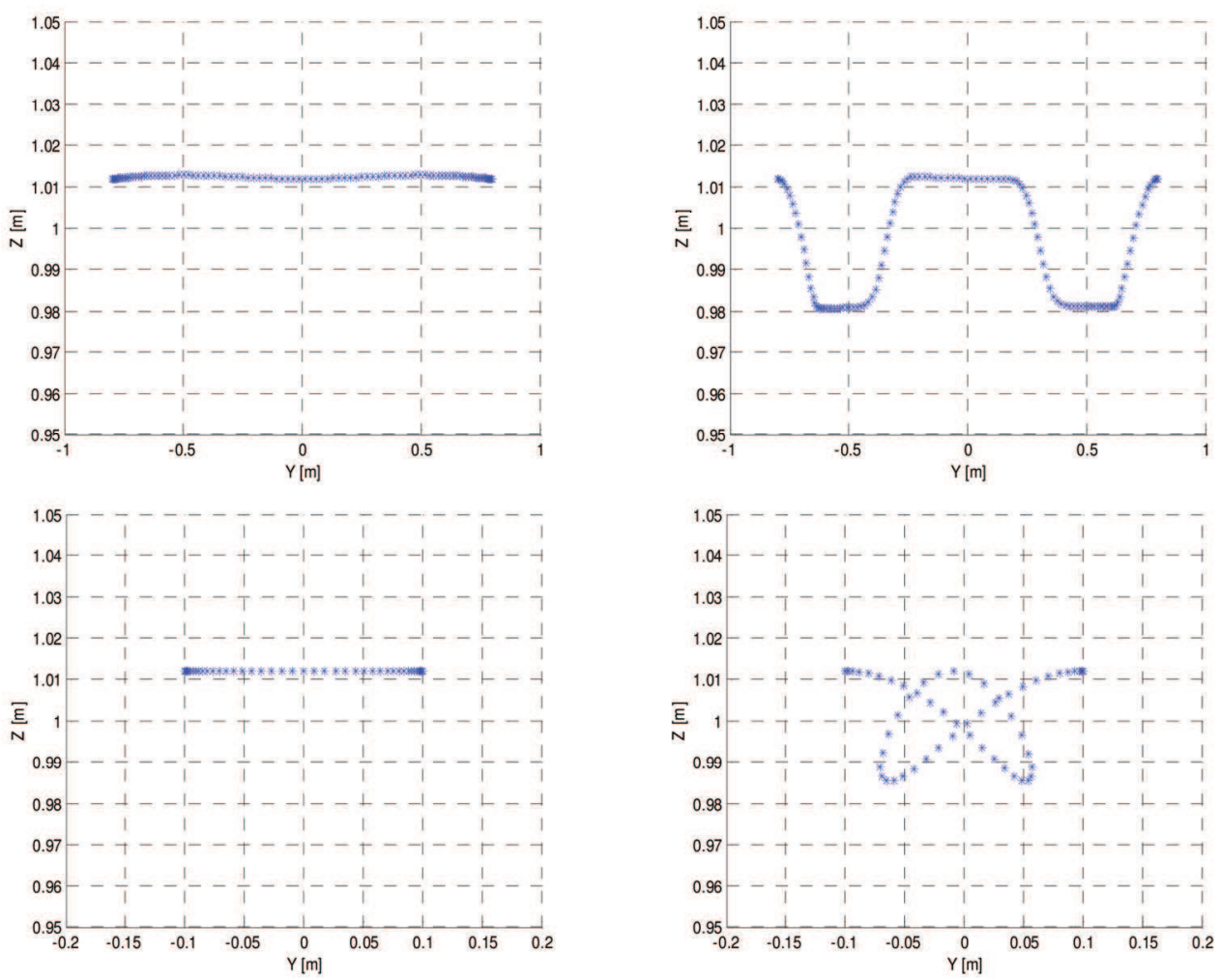

Figure 14. Two test-trajectories. Left: non-compensated motion. Right: compensated motion

\subsection{Acceleration Excitation}

Now, we proceed to evaluate the excitation effects of acceleration in the compensation of sways. Recalling the Fig. 15, in order to compensate the swing effects induced by the driving accelerations, additional acceleration excitations are applied [Fig. 15 (right)] at the changing phases from the reference acceleration profile [Fig. 15 (left)].

As described in section 2, the work from [Mita \& Kanai, 1997] reveal that it is possible to obtain a swing-free motion at the end of the desired trajectory by constraining the velocity profile. This means that an optimal time swing-free motion could be achieved using a sequence of constant acceleration pulses to cancel undesirable swing effects in transportation of suspended objects. The similar principle and resulting effects can be observed with the results obtained in this study.

However, a proper modification of the reference motion using the ACP is more efficient and convenient, because of the simplicity of its computing algorithm and great facility to be implemented into the robot system. Furthermore, there is no need of pre-designing the velocity profile, where the knowledge of different phase-time instances are necessarily to be known in advance. 


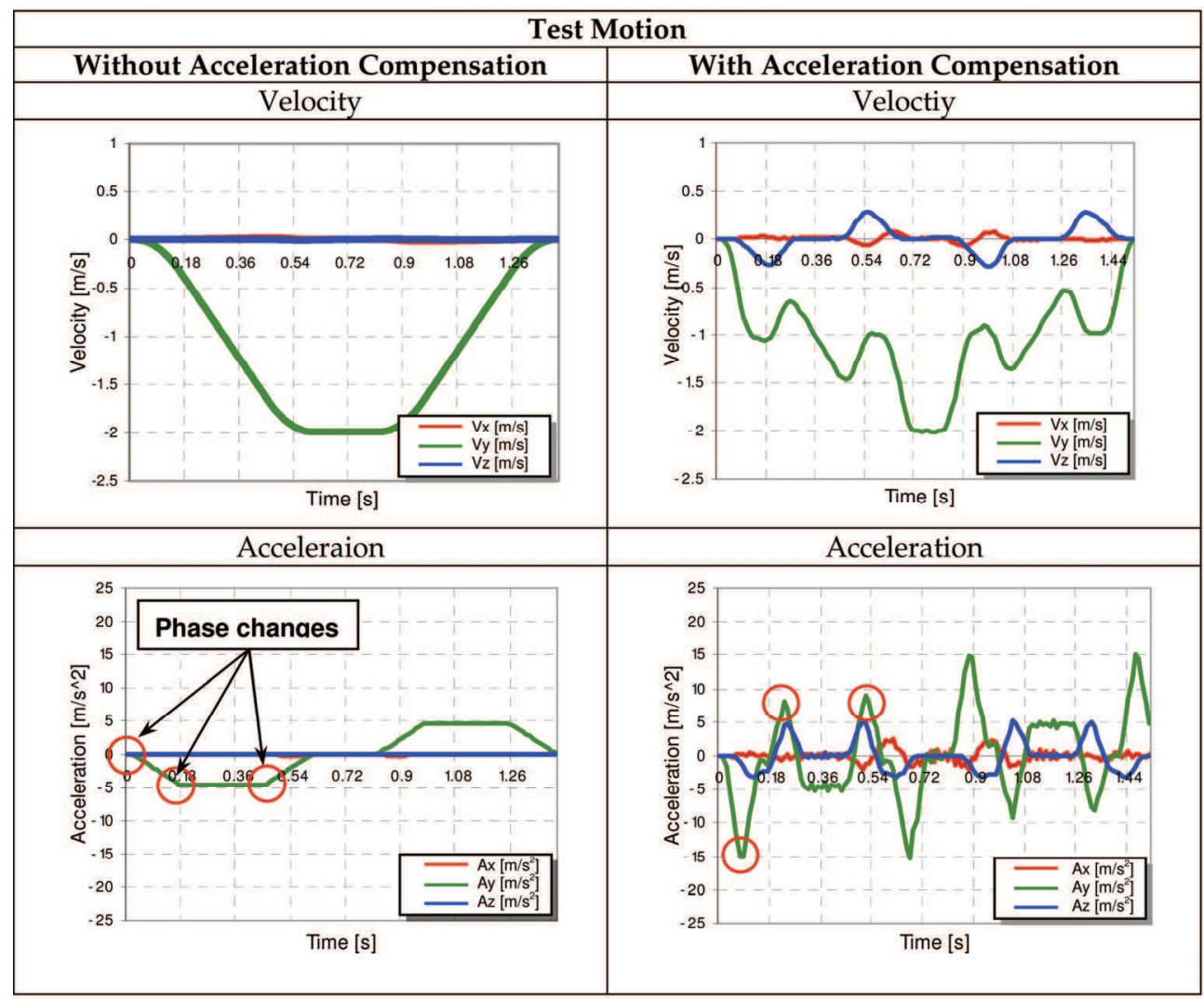

Figure 15. Velocity and acceleration profiles from the test motion. Non-compensated motion vs. compensated motion

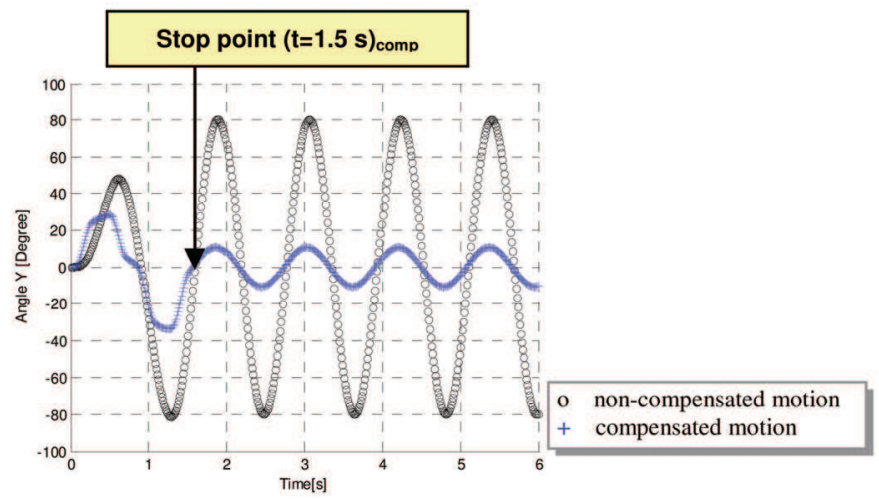

Figure 16. Test motion. Swing angles in indirection. Non-compensation vs. compensation 


\subsection{Integrating Physics Engine into the Simulation Environment}

Before the execution of the compensated motion with the real robot system, open-source physics engine 'Bullet' has been incorporated into our software simulation system, in order to observe in advance the nonlinear behaviours of those test-objects with dynamical characteristics, to avoid in this way, the causing of any possible hazard [Fig. 17].
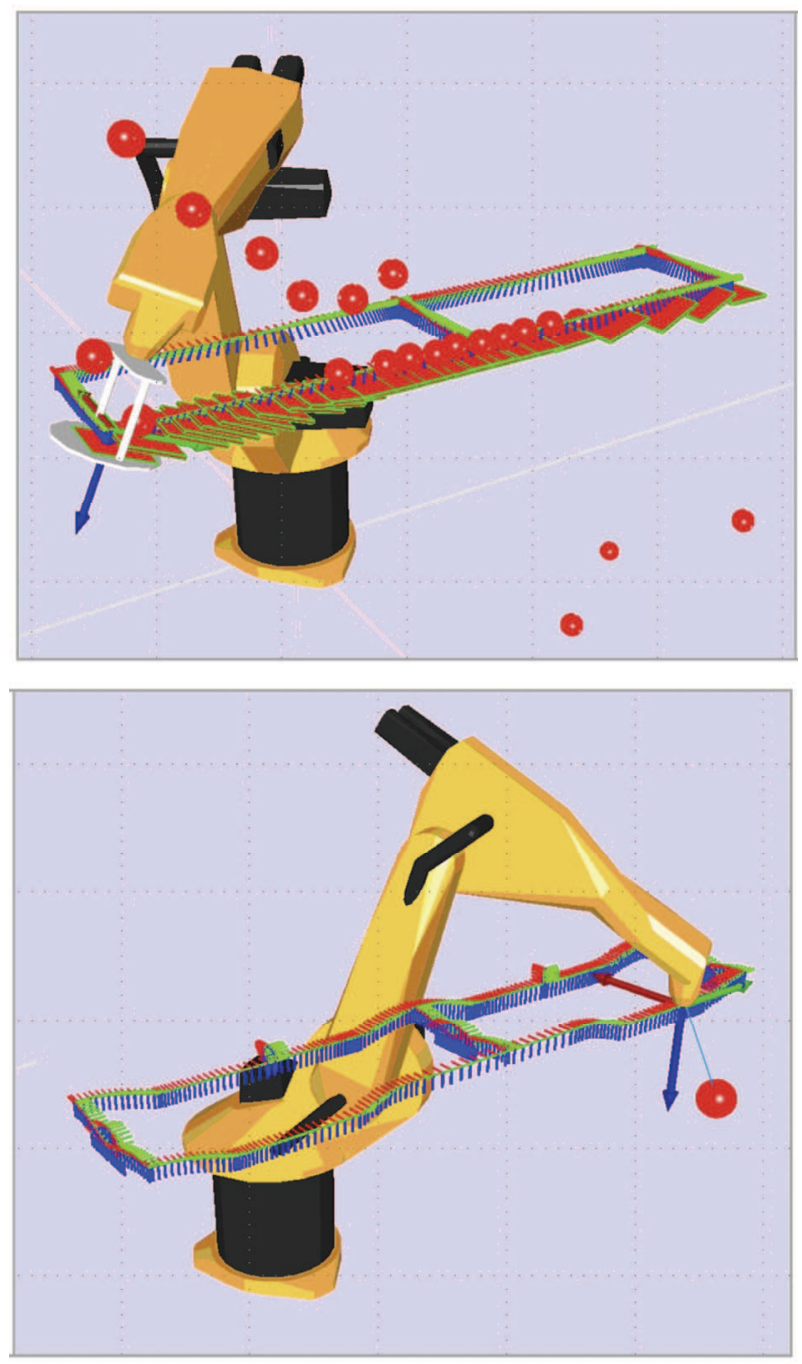

Figure 17. Software simulation using physics engine 'Bullet'

\section{Conclusion}

The acceleration compensation principle (ACP) proved to be beneficial and powerful for compensating side effects caused by acceleration, commonly produced on handling objects 
with dynamical constraints. In order to deal with different kinds of handling problems caused by highly accelerated motions, new efficient solutions based on this principle have been established. Gentle robotic handling and time optimized movements are considered as two main research objectives. Beyond the achievement of optimal cycle time and the avoidance of undesirable collisions, the proposed algorithms are independent of the physical aspects of the object, such like shape, material, weight and the size. As main part of the experimental test-bed, several serial robot manipulators have been utilized with diverse test objects, to verify the feasibility and effectiveness of the proposed approaches. Three different kinds of problem-scenarios dealing with robotic handling are investigated within the scope of this study: minimization of undesirable large shear forces, fluid sloshing and residual swing oscillations produced in suspended objects.

As final conclusion, the acceleration compensation method with its simplicity and robustness, has demonstrated its potential with satisfactory results. As future work, the incorporation of a compensation on the fly will be suggested as a potential improvement of the proposed methods, in order to make them more applicable and efficient for the real industrial applications.

\section{References}

N. Blazicevic and B. Novakovic (1996). Control of a rotary crane by a combined profile of speeds, Strojarstvo, v. 38, no. 1, pp. 13-21.

S. J. Chen, H. Worn, U. Zimmermann, R Bischoff (2006). Gentle Robotic Handling Adaptation of Gripper-Orientation To Minimize Undesired Shear Forces Proceedings of the 2006 IEEE International Conference on Robotics and Automation (ICRA), Orlando, Florida, pp. 309-314.

S. J. Chen, B. Hein, and H. Worn (2006). Applying Acceleration Compensation to Minimize Liquid Surface Oscillation, - Australasian Conference on Robotics and Automation, December, Auckland, New Zealand.

S. J. Chen, B. Hein, and H. Worn (2007). Swing Attenuation of Suspended Objects Transported by Robot Manipulator Using Acceleration Compensation, Proceedings of the 2007 IEEE/RSJ International Conference on Intelligent Robots and Systems (IROS), October, San Diego, USA.

A.X. Dang (2002). PHD Thesis: Theoretical and Experimental Development of an Active Acceleration Compensation Platform Manipulator for Transport of Delicate Objects, Georgia Institute of Technology.

A.X. Dang and I. Ebert-Uphoff (2004), Active Acceleration Compensation for Transport Vehicles Carrying Delicate Objects, IEEE Trans, on Robotics, Vol. 20, No. 5.

M.W. Decker, A.X. Dang, I. Ebert-Uphoff (2001). Motion Planning for Active Acceleration Compensation, Proceedings of the 2001 IEEE International Conference on Robotics $\mathcal{E}$ Automation, Seoul, Korea.

J. Feddema, C. Dohrmann, G. Parker, R. Robinett, V. Romero and D. Schmitt (1996). Robotically Controlled Slosh-Free Motion of an open Container of Liquid, IEEE International Conf. On Robotics and Automation, Minneapolis, Minnesota.

J. Feddema, C. Dohrmann, G. Parker, R. Robinett, V. Romero and D. Schmitt (1997). A Comparison of Maneuver Optimization and Input Shaping Filters for Robotically Controlled Slosh-Free Motion of an Open Container of Liquid, Proceedings of the American Control Conference, Albuquerque, New Mexico. 
J. T. Feddema et al.(1997), Control for slosh-free motion of an open container, IEEE Contr. Syst. Mag., vol. 17, no. 1, pp. 29-36, Feb.

R. Graf, R. Dillmann (1997). Active acceleration compensation using a Stewart-platform on a mobile robot, Proceedings of the 2nd Euromicro Workshop on Advanced Mobile Robots, EUROBOT '97, Brescia, Italy, pp. 59-64.

R. Graf, R. Dillmann (1999). Acceleration Compensation Using a Stewart-Platform on a Mobile Robot, Proceedings of the 3rd European Workshop on Advanced Mobile Robots, EUROBOT '99, Zurich, Switzerland.

R. Graf (2001). PHD Thesis: Aktive Beschleunigungskompensation mittels einer StewartPlattform auf einem mobilen Roboter, Universitat Karlsruhe. ISBN: 3-935363-37-0.

F. Crasser, A. D'Arrigo, S. Colombi, and A. Rufer (2002). Joe: A mobile, inverted pendulum, IEEE Trans. Ind. Electron., vol. 49, no. 1, pp. 107-114.

M. Hamaguchi, K. Terashima, and H. Nomura (1994). Optimal control of liquid container transfer for several performance specifications, /. Adv. Automat. Technol, vol. 6, pp. 353-360.

M. Kaneko, Y. Sugimoto, K. Yano (2003). Supervisory Control of Pouring Process by TiltingType Automatic Pouring Robot, Proceedings of the 2003 lEEE/RSf International Conference on Intelligent Robots and Systems, Las Vegas, Nevada.

R. Kolluru, K.P. Valavanis, S.A. Smith and N. Tsourveloudis (2000). Design Fundamentals of a Reconfigurable Robotic Gripper System, IEEE Transactions on Systems, Man, and Cybernetics-Part A: Systems and Humans, Vol. 30, No.2.

T. Lauwers, G. Kantor and R. Hollis (2005). One is Enough!, 12th Int'l Symp. On Robotics Research. San Francisco.

Y. Lerner and N. Laukhin (2000). Development trends in pouring technology, Foundry Trade \}., vol. 11, pp. 16-21.

W. Linsay (1983). Automatic pouring and metal distribution system, Foundry Trade \}., pp. 151-165.

T. Mita, and T. Kanai (1979). Optimal Control of the Crane System Using the Maximum Speed of the Trolley, Trans. Soc. Instrument. Control Eng. (Japan), 15, pp. 833-838.

Y. Noda, K. Yano, K. Terashima (2002). Tracking Control with Sloshing-Suppression of SelfTransfer Ladle to Continuous-Type Mold Line in Automatic Pouring System, Proc. of SICE Annual Conference (2002), Osaka.

Y. Noda, K. Yano, S. Horihata and K. Terashima (2004). Sloshing suppression control during liquid container transfer involving dynamic tilting using wigner distribution analysis, 43th IEEE Conference on Decision and Control, Atlantis, Bahamas.

G. G. Parker, B. Petterson, C. R. Dohrmann, and R. D. Robinett III (1995).Vibration Suppression of Fixed-Time Jib Crane Maneuvers, Sandia National Laboratories report SAND95-0139C.

Y. Sakawa and Y. Shindo (1982). Optimal control of container cranes, Automatica, v. 18, no. 3, pp. 257-266.

Segway Human Transporter (2004). [Online]. Available: http:/ / www.segway.com

N. C. Singer and W. P. Seering (1990). Preshaping Command Inputs to Reduce System Vibration, ASME Journal of Dynamic Systems, Measurement,and Control.

W. E. Singhose, L. J. Porter, and W. P. Seering (1997). Input Shaped Control of a Planar Gantry Crane with Hoisting, American Control Conference, Albuquerque, NM. 
O. J. M. Smith (1957). Posicast Control of Damped Oscillatory Systems, Proc. of the IRE, pp. 1249-1255.

G. P. Starr (1985). Swing-Free Transport of Suspended Objects with a Path-Controlled Robot Manipulator, ASME Journal of Dynamic Systems, Measurement, and Control, v. 107.

K. Terashima and K. Yano (2001). Sloshing analysis and suppression control of tilting-type automatic pouring machine, IF AC J. Contr. Eng. Practice, vol. 9, no. 6, pp. 607-620.

N.C. Tsourveloudis, R. Kolluru, K.P. Valavanis and D. Gracanin (2000). Suction Control of a Robotic Gripper: A Neuro-Fuzzy Approach, Journal of Intelligent and Robotic System 27: 215-235.

K. Yano and K. Terashima (2001). Robust Liquid Container Transfer Control for Complete Sloshing Suppression, IEEE Transactions, on Control Systems Technology, Vol. 9,No. 3. 


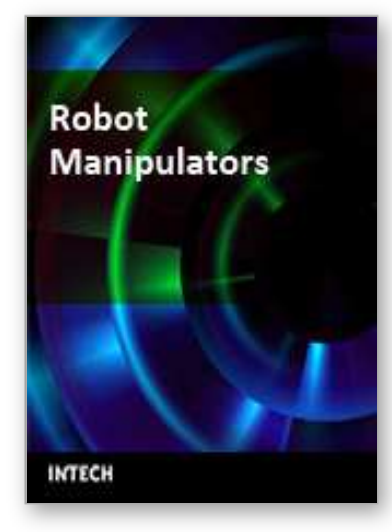

\author{
Robot Manipulators \\ Edited by Marco Ceccarelli
}

ISBN 978-953-7619-06-0

Hard cover, 546 pages

Publisher InTech

Published online 01, September, 2008

Published in print edition September, 2008

In this book we have grouped contributions in 28 chapters from several authors all around the world on the several aspects and challenges of research and applications of robots with the aim to show the recent advances and problems that still need to be considered for future improvements of robot success in worldwide frames. Each chapter addresses a specific area of modeling, design, and application of robots but with an eye to give an integrated view of what make a robot a unique modern system for many different uses and future potential applications. Main attention has been focused on design issues as thought challenging for improving capabilities and further possibilities of robots for new and old applications, as seen from today technologies and research programs. Thus, great attention has been addressed to control aspects that are strongly evolving also as function of the improvements in robot modeling, sensors, servo-power systems, and informatics. But even other aspects are considered as of fundamental challenge both in design and use of robots with improved performance and capabilities, like for example kinematic design, dynamics, vision integration.

\title{
How to reference
}

In order to correctly reference this scholarly work, feel free to copy and paste the following:

Suei Jen Chen (2008). Gentle Robotic Handling Using Acceleration Compensation, Robot Manipulators, Marco Ceccarelli (Ed.), ISBN: 978-953-7619-06-0, InTech, Available from:

http://www.intechopen.com/books/robot_manipulators/gentle_robotic_handling_using_acceleration_compensa tion

\section{INTECH}

open science | open minds

\author{
InTech Europe \\ University Campus STeP Ri \\ Slavka Krautzeka 83/A \\ 51000 Rijeka, Croatia \\ Phone: +385 (51) 770447 \\ Fax: +385 (51) 686166 \\ www.intechopen.com
}

\author{
InTech China \\ Unit 405, Office Block, Hotel Equatorial Shanghai \\ No.65, Yan An Road (West), Shanghai, 200040, China \\ 中国上海市延安西路65号上海国际贵都大饭店办公楼405单元 \\ Phone: $+86-21-62489820$ \\ Fax: $+86-21-62489821$
}


(C) 2008 The Author(s). Licensee IntechOpen. This chapter is distributed under the terms of the Creative Commons Attribution-NonCommercialShareAlike-3.0 License, which permits use, distribution and reproduction for non-commercial purposes, provided the original is properly cited and derivative works building on this content are distributed under the same license. 